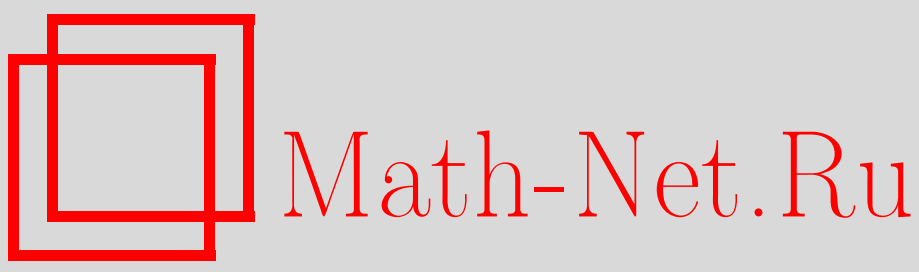

А. А. Туганбаев, Прямые суммы дистрибутивных модулей, Матем. сб., 1996, том 187, номер 12, 137-156

DOI: https://doi.org/10.4213/sm181

Использование Общероссийского математического портала Math-Net.Ru подразумевает, что вы прочитали и согласны с пользовательским соглашением

http://www . mathnet.ru/rus/agreement

Параметры загрузки:

IP : 34.239 .49 .27

26 апреля 2023 г., 13:19:34 
УДК 512.55

\author{
А.А. Туганбаев
}

\title{
Прямые суммы дистрибутивных модулей
}

\begin{abstract}
Модуль назьвается дистрибутивным, если решетка всех его подмодулей дистрибутивна. Прямая сумма дистрибутивных модулей называется полудисmрибутивным модулем. Доказано, что первичный радикал кольца эндоморфизмов конечной прямой суммы дистрибутивных модулей содержит все односторонние нильидеалы кольца эндоморфизмов этого модуля. Полупервичное кольцо с условием максимальности для правых аннуляторов, разлагающееся в прямую сумму дистрибутивных правых идеалов, является конечным прямым произведением первичных колец.

Библиограффия: 12 названий.
\end{abstract}

\section{Введение}

Все кольца предполагаются ассоциативньми и, за исключением особо оговоренных случаев, с ненулевой единицей. Слова типа "артиново кольцо" означают, что соответствующие условия выполнены справа и слева. Через $\operatorname{Lat}(M)$ и $\operatorname{End}(M)$ обозначаются, соответственно, решетка всех подмодулей и кольцо эндоморфизмов модуля $M$. Модуль $M$ называется дистрибутивным (иепнымм), если решетка $\operatorname{Lat}(M)$ дистрибутивна (является цепю). Первичный радикал дистрибутивного справа кольца $A$ содержит все правые или левые нильидеалы кольца $A$ (для двусторонних идеалов это доказал автор в [1], а для односторонних нильидеалов Мазурек и Пучиловский в [2]). Прямая сумма дистрибутивных (цепных) модулей называется полудистрибутивнылм (полуцепнылм) модулем.

Примерами полудистрибутивных колец являются все полуцепные кольца (например, кольца верхнетреугольных матриц над телом), все дистрибутивные кольца (например, все факторкольца прямых произведений тел, все коммутативные кольца Безу и коммутативные кольца единичной слабой глобальной размерности) и кольца матриц над коммутативньми полунаследственными кольцами (например, кольцо матриц над кольцом целых чисел).

Данная работа посвящена исследованию полудистрибутивных колец и модулей. Из результатов работы выделим теоремы 1 и 2 . В теореме 1 , в частности, доказано, что первичный радикал кольца $R$ эндоморфизмов конечной прямой суммы дистрибутивных модулей содержит все односторонние нильидеалы кольца $R$. Поэтому первичньй радикал полудистрибутивного справа кольца содержит все односторонние нильидеалы этого кольца, что перекрывает упомянутый результат Мазурека и Пучиловского. В теореме 2, в частности, доказано, что полудистрибутивное справа полупервичное кольцо с условием максимальности для правых аннуляторов разлагается в конечное прямое произведение первичных колец.

Отметим также, что в работе приведен контрпример к некоторым утверждениям работы [3] (см. замечание 6). 
Доказательство теорем разбито в работе на ряд утверждений, некоторые из которых представляют самостоятельный интерес. Напомним ряд определений и обозначений. Через $r_{A}(N)$ обозначается аннулятор в кольце $A$ подмножества $N$ правого модуля $M_{A}$, причем индекс может опускаться, когда ясно, какое кольцо имеется в виду. Через $\operatorname{Sing}(M)$ обозначается сингулярныи й подмодуль модуля $M_{A}$ над кольцом $A$ (т.е. множество всех таких элементов $m \in M$, что $r(m)$ - сушественный правый идеал кольца $A$ ). Подфактором называется подмодуль фактормодуля. Модуль $M_{A}$ называется несингулярныцм (сингулярным), если $\operatorname{Sing}(M)=0$ $(\operatorname{Sing}(M)=M)$. Если $A$ - кольцо, то $\operatorname{Sing}\left(A_{A}\right)$ - идеал кольца $A$, который называется правым.м сингулярнымм идеалом кольца $A$. Модуль называется равномерныцм, если любые его два ненулевых подмодуля имеют ненулевое пересечение. Полуравномерным модулем называется прямая сумма равномерных модулей. Модуль, не содержаший бесконечных прямых сумм ненулевых подмодулей, назьвается конечномернылм. Класс конечномерных модулей совпадает с классом существенных расширений конечных прямых сумм равномерных модулей. Конечномерное справа кольцо с условием максимальности для правых аннуляторов называется правылм кольцом Голди. Подмодуль $N$ модуля $M$ называется замкнутылм в модуле $M$, если $N$ совпадает с любым подмодулем модуля $M$, являюшимся существенным расширением модуля $N$. Кольцо называется нормальныцм, если все его идемпотенты центральны. Кольцо без ненулевых нильпотентных элементов называется редущированным кольцом. Кольцо $A$ называется риккартовым справа, если оно удовлетворяет следующим двум эквивалентным условиям:

(1) все главные правые идеалы проективны как правые $A$-модули;

(2) правьй аннулятор любого элемента кольца $A$ порождается (как правый идеал) идемпотентом.

Идемпотент $e$ кольца $A$ называется локальным, если $e A e$ - локальное кольцо. Полусовершенность кольца $A$ равносильна тому, что его единица разлагается в сумму локальных ортогональных идемпотентов. Кольцо $A$ называется регулярным (строго регулярным ), если для любого элемента $a \in A$ найдется такой элемент $b \in A$, что $a=a b a\left(a=a^{2} b\right)$. Зафиксируем разложение $1=\sum_{i=1}^{n} e_{i}$ единицы кольца $A$ в сумму ортогональных идемпотентов $e_{i}$. Кольцо $A$ называется кусочно целостным (относительно $\left.\left\{e_{1}, \ldots, e_{n}\right\}\right)$, если оно удовлетворяет следующим трем эквивалентным условиям (см. [4]):

(1) для всех $e_{i}$ и $e_{k}$ каждый ненулевой гомоморфизм $e_{i} A \rightarrow e_{k} A$ является мономорфизмом;

(2) для всех $e_{i}$ каждый ненулевой гомоморфизм $e_{i} A \rightarrow A_{A}$ является мономорфизмом;

(3) $a b \neq 0$ для всех ненулевых элементов $a \in e_{j} A e_{i}, b \in e_{i} A e_{k}$ и для любых $e_{i}, e_{j}, e_{k}$.

Поскольку (3) симметрично, то (1) и (2) можно заменить на их левосторонние аналоги. Собственный идеал $B$ кольца $A$ будем называть вполне первичным (nервичным; несингулярно первичным; несингулярно полупервичным; кусочно целостным относительно $\left\{e_{1}, \ldots, e_{n}\right\}$ ), если $A / B$ - область (первичное кольцо; несингулярное справа первичное кольцо; несингулярное справа полупервичное кольцо; кусочно целостное относительно естественных образов $\left\{e_{1}, \ldots, e_{n}\right\}$ в $A / B$ кольцо). Несингулярно первичньй (вполне первичный; несингулярно по- 
лупервичный) идеал кольца $A$, не содержащий других несингулярно первичных (вполне первичных; несингулярно полупервичных) идеалов кольца $A$, называется минимальным несингулярно первичным идеалом (минимальным вполне первичным идеалом; минимальным несингулярно полупервичным идеалом). В полупервичном кольце $A$ правый и левый аннуляторы любого идеала совпадают; поэтому идеал кольца $A$ называется аннуляторным идеалом, если он является правым или левым аннулятором некоторого идеала кольца $A$. Будем говорить, что индекс кольца $A$ не превосходит натурального числа $n$, если $a^{n}=0$ для любого нильпотентного элемента $a \in A$. Модуль $M$ будем для краткости назьвать нильпотентно-инвариантным, если каждьй его нильпотентньй эндоморфизм переводит в себя все подмодули модуля $M$. Модуль $M_{A}$ называется вполне nервичным. если $r(m)=r(M)$ для любого ненулевого элемента $m \in M$. Если $B-$ идеал кольца $A$, то $(A / B)_{A}$ - вполне первичный модуль $\Longleftrightarrow B$ - вполне первичный идеал кольца $A$.

\section{§1. Кольца эндоморфизмов}

ЛЕмма 1. Пусть $M$ - нильпотентно-инвариантный модуль, $N$ - подмодуль модуля $M, T$ - множество всех нильпотентных әндоморфизмов модуля $M$.

(1) Eсли $f_{i}, g_{j} \in T$, где $1 \leqslant i, j \leqslant n, u\left(f_{1} f_{2} \cdots f_{n}\right)(N)=0$, mo $\left(f_{1} g_{1} f_{2} g_{2} \cdots f_{n} g_{n}\right)(N)=0$.

(2) Ecлuf,g $\in$ Tu $f^{m}(N)=g^{n}(N)=0, m o(f+g)^{m+n}(N)=0$.

(3) Eсли $f \in T, T_{1}$ - подкольио в $\operatorname{End}(M)$, порожденное единицей и множсеством $T$, причем $f^{n}(N)=0$, то $\left(f T_{1}\right)^{n}(N)=0$.

(4) Мнохсество $T$ является нильподкольцом кольца $\operatorname{End}(M)$.

(5) Нилькольцо T совпадает с суммой своих нильпотентных идеалов.

(6) Первичный радикал $P$ кольца $\operatorname{End}(M)$ содерэсит все правые нильидеальь и все левье нильидеаль кольца $\operatorname{End}(M)$.

(7) Если $f \in T$, то $\operatorname{Ker}(f)$ - существенньй подмодуль модуля $M$ и $f(M)-$ сингулярный модуль.

ДоказАТЕльство. Пункт (1) проверяется непосредственно; (2) и (3) следуют из (1); (4) следует из (1) и (2); (5) следует из (4) и (3). Докажем (6). Пусть $R \equiv \operatorname{End}(M), D$ - идеал кольца $R$, порожденньй произвольным правым нильидеалом $E$ кольца $R$. Из равенства $(x y)^{n+1}=x(y x)^{n} y$ следует, что $D$ - нильидеал кольца $R$. По (5) $D=\sum_{j \in J} T_{j}$, где $\left\{T_{j}\right\}_{j \in J}$ - некоторое множество нильпотентных идеалов нилькольца $D$. Идеал $D_{j}$ кольца $R$, порожденный множеством $T_{j}$, лежит в $P$, поскольку является нильпотентньм идеалом. (Это вытекает из следующего утверждения [5, лемма 1.1.5]: если $D$ - идеал кольца $R, T$ - нильпотентньй идеал кольца (не обязательно с единицей) $D$, то идеал кольца $R$, порожденнњй множеством $T$, нильпотентен.) Так как $D=\sum_{j \in J} T_{j}$ и $D$ - идеал кольца $R$, то $D=\sum_{j \in J} D_{j} \subseteq P$. Тогда $E \subseteq D \subseteq P$. Аналогично, $P$ содержит все левые нильидеалы кольца $R$.

(7). Пусть $N$ - такой подмодуль модуля $M$, что $N \cap \operatorname{Ker}(f)=0$. Допустим, что $N \neq 0$. Так как $M$ - нильпотентно-инвариантньй модуль, то $0 \neq f^{n}(N) \subseteq N$ для всех $n$, что противоречит нильпотентности эндоморфизма $f$. Поэтому $M-$ 
сушественное расширение модуля $\operatorname{Ker}(f)$. Тогда $M / \operatorname{Ker}(f)$ - сингулярный модуль, откуда $f(M)$ - сингулярный модуль.

ЛЕмма 2. Пусть $M$ - несингулярный нильпотентно-инвариантный мо$\partial y л ь$.

Тогда $\operatorname{End}(M)-$ редуцированное кольцо. Если, кроме того, $\operatorname{End}(M)-$ первичное кольио, то $\operatorname{End}(M)$ - область.

ДоказАтельство. Редуцированность $\operatorname{End}(M)$ следует из леммы 1(7). Второе утверждение следует из того, что редуцированное первичное кольцо является областью (см. [5, с. 288]).

Лемма 3. Пусть е - ненулевой идемпотент кольца $A$.

(1) Пусть $M_{A}-$ правый $A$-модуль. Правилом $\varphi(N)=N$ е задается сюрьективный решеточный гомоморфизм $\varphi: \operatorname{Lat}(M) \rightarrow \operatorname{Lat}\left((M e)_{e A e}\right)$, причем если $M_{A}=\bigoplus_{j \in J} M_{j}$, то $M e_{e A e}=\bigoplus_{j \in J} M_{j} e$.

(2) Если $F$ - идеал кольца $A, e \in A \backslash F, h: A \rightarrow A / F$ - естественный әпиморфизм, то существует естественный кольцевой изоморфизм $h(e) h(A) h(e) \rightarrow e A e / e F e$.

(3) Eсли $N$ - идеал кольца еAe, mо $N=e(A N A) e$.

(4) Если $A$ - кольцо с условием максимальности для правых (левых) аннуляторов, то еАе - кольцо с условием максимальности для правых (левьх) аннуляторов.

(5) Если $A / F$ - факторкольцо с условием максимальности для правых аннуляторов, то еАе/еFе - кольцо с условием максимальности для правъх аннуляторов.

(6) Если модуль $M_{A}$ дистрибутивен (полудистрибутивен; нётеров; имеет размерность Крулля), то модуль (Ме) еАе дистрибутивен (полудистрибутивен; нётеров; имеет размерность Крулля).

(7) Если модуль еА дистрибутивен (полудистрибутивен; нётеров; имеет размерность Крулля), то кольцо еАе дистрибутивно справа (полудистрибутивно справа; нётерово справа; имеет правую размерность Крулля).

(8) Если $P$ - первичный радикал кольца $A$, то еPе - первичныцй радикал кольиа еАe.

(9) Если F - первичный идеал кольца A, не содержащий е, то еFe - собственный первичный идеал кольца еАе.

(10) Если $A$ - полупервичное (первичное; простое) кольчо, то еАе - полупервичное (первичное; простое) кольцо.

(11) Пусть $F, G$ - не сравнимье по включению первичные идеаль кольца $A$ и идемпотент е не лежит в $F+G$. Тогда еFe, eGe - не сравнимье по включению первичные идеаль кольца еАе и еFe $+e G e \neq e A e$.

(12) Если n-натуральное число и все п-порожденнье правые идеаль кольца А проективны, то все п-порожденные правые идеаль кольца еАе проективны.

(13) Пусть $A$ - первичное кольцо. Тогда eAe - область

$\Longleftrightarrow$ любой ненулевой эндоморфизм правого идеала еА является мономорфизмом 
$\Longleftrightarrow$ любой ненулевой эндоморфизм каждого левого идеала Ае является мономорфизмом.

ДокАЗАТЕльство. Пункты (1), (2) и (3) проверяются непосредственно; (4) следует из того, что $r_{e A e}(B)=e A e \cap r_{A}(B)$ для любого подмножества $B$ в $e A e$. (5) следует из (2) и (4); (6) следует из (1); (7) следует из (6).

(8). Первичньй радикал $P$ кольца $A$ совпадает со множеством всех строго нильпотентных в $A$ элементов. (Элемент а кольца $A$ называется строго нильпотентным в $A$, если все члены любой такой последовательности $\left\{a_{n}\right\}$, что $a_{1}=a$, $a_{n+1} \in a_{n} A a_{n}$, равны нулю, начиная с некоторого номера.) Все элементы из $e P e$ строго нильпотентны в $A$, поскольку $e P e \subseteq P$. Остается доказать, что если $a \in A$ и еае строго нильпотентный в $е A e$ элемент, то элемент еае строго нильпотентен в $A$. Это вытекает из того, что если $a_{1}=e a e, a_{n+1} \in a_{n} A a_{n}$, то все элементы $a_{n}$ лежат в $e A e$.

(9). Пусть $F$ - первичный идеал. Так как $e \in A \backslash F \subseteq A \backslash e F e$, то $e F e \neq e A e$. Допустим, что $e F e$ не является первичным идеалом кольца $e A e$. Тогда из (3) вытекает существование таких идеалов $M, N$ кольца $A$, что $e M e N e \subseteq e F e \subseteq F$. Тогда идеал $(A e A) M(A e A) N(A e A)$ лежит в первичном идеале $F$. Поэтому либо $M \subseteq F$, либо $N \subseteq F$. Тогда либо $e M e \subseteq e F e$, либо $e N e \subseteq e F e$.

Пункт (10) следует из (3),(8) и (9).

(11). Так как $e \in A \backslash(F+G)$, то $e F e+e G e \neq e A e$. По (9) eFe и $e G e-$ первичные идеалы кольца $e A e$. Допустим, что $e F e \subseteq e G e$. Тогда идеал $(A e A) F(A e A)$ лежит в первичном идеале $G$, и получаем противоречие. Аналогично, невозможно включение $e F e \supseteq e G e$.

(12). Пусть $B=\sum_{i=1}^{n} b_{i} e A e-n$-порожденньй правый идеал кольца $e A e$, $\bar{B} \equiv B+B A(1-e)$. Тогда $\bar{B}=\sum_{i=1}^{n} b_{i} A-n$-порожденный правый идеал кольца $A$. Пусть $F_{A}-$ прямая сумма $n$ экземпляров модуля $e A$. Через $e_{i}$ обозначим элемент-строку модуля $F$, у которого на $i$-м месте стоит элемент $e$, а на остальных местах - нули. Сушествует такой эпиморфизм $h: F \rightarrow \bar{B}$, что $h\left(e_{i}\right)=b_{i}$ для всех $i$. Так как по условию $\bar{B}_{A}-$ проективный модуль, то существует такой мономорфизм $g: \bar{B} \rightarrow F$, что $h g$ тождественно действует на $\bar{B}$. Тогда $F=\operatorname{Im}(g) \oplus \operatorname{Ker}(h)$. Домножая это равенство на $e$, получаем равенство $(e A e)^{(n)}=\operatorname{Im}(g) e \oplus \operatorname{Ker}(h) e$. Так как $\operatorname{Im}(g) e=B$, то $B_{e} A e^{-}$проективньй модуль.

(13). Достаточно доказать первую эквивалентность. Импликация $=$ очевидна. Докажем $\Longrightarrow$. Так как $e A e$ можно естественно отождествить с $\operatorname{End}(e A)$, то достаточно привести к противоречию следующее утверждение: существуют такие ненулевые элементы $a=e a e \in e A e$ и $b=e b \in e A$, что $e a e b=0$. Тогда $(e a e)(e b A e)=0$. Так как $e A e-$ область и $е a e \neq 0$, то $e b A e=0$. Тогда $A e b A e A=0$. Поскольку $A$ первично, то либо $e b=0$, либо $e=0$, что невозможно.

Лемма 4. Пусть $1=e_{1}+\cdots+e_{n}-$ разложсение единици кольиа $A$ в сумму ненулевых ортогональных идемпотентов, $P$ - первичныи радикал кольиа $A$, $N-$ правый идеал кольца $A, M_{A}-$ правый $A$-модуль.

(1) Если $N$ - правый нильидеал кольца $A$, то $e_{i} N e_{i}-$ правый нильидеал кольиа е $e_{i} A e_{i}$ для любого $i$.

(2) $E$ сли $e_{i} N e_{i}=0$ для всех $i$, mо $N^{n}=0$.

(3) Если для любого і правый идеал $e_{i} N e_{i}$ кольиа $e_{i} A e_{i}$ лежит в первичном радикале $P_{i}$ кольиа $e_{i} A e_{i}$, то $N$ лежств в $P$. 
(4) Если для любого $і$ первичный радикал кольца $e_{i} A e_{i}$ содержит все правые нильидеальи (все левье нильидеаль) кольца $e_{i} A e_{i}$, то $P$ содержит все правье нильидеаль (все левье нильидеаль) кольца $A$.

(5) Если для любого $i$ кольцо $e_{i} A e_{i}$ полупервично, то $P^{n}=0$.

(6) Если $i \in\{1, \ldots, n\}$ и ае $e_{i} \in A e_{i}$, то для включения ае $i \in \operatorname{Sing}\left(A_{A}\right)$ необходимо и достаточно, чтобъ существовал такой существенный подмодуль $B_{i}$ модуля $e_{i} A$, что ае ${ }_{i} B_{i}=0$.

(7) Если а - әлемент кольца $A$, то для включения $a \in \operatorname{Sing}\left(A_{A}\right)$ необходимо и достаточно, чтобь для любого е ${ }_{i}$ существовал такой существенный подмодуль $B_{i}$ модуля $e_{i} A$, что ае ${ }_{i} B_{i}=0$.

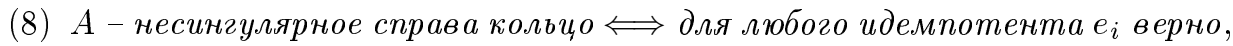
что $а B \neq 0$ для каждого ненулевого әлемента $a \in A_{i}$ и для любого существенного подмодуля $B$ модуля $e_{i} A$.

(9) Любой правый модуль $X_{A}$ над кольцом $A$ является как абелева группа прямой суммой правых $e_{i} A e_{i}$-модулей $X e_{i}$.

(10) Если $F$ и $G-$ подмодули модуля $M_{A}$, mo $F+G=\sum_{i=1}^{n}\left(F e_{i}+G e_{i}\right)$, $F \cap G=\sum_{i=1}^{n} F e_{i} \cap G e_{i}$.

(11) Дистрибутивность модуля $M_{A}$ равносильна дистрибутивности всех $e_{i} A e_{i}$-модулей $M e_{i}$.

(12) $A$ - кусочно иелостное (относительно $\left.\left\{e_{1}, \ldots, e_{n}\right\}\right)$ кольцо $\Longleftrightarrow a b \neq 0$ для всех ненулевых әлементов $a \in A e_{i}, b \in e_{i} A$ и для каждого $e_{i}$.

(13) Пусть $F$ - первичный идеал кольца $A$. Тогда $F$ - кусочно челостный идеал $\Longleftrightarrow$ для любого $e_{i} \in A \backslash F$ идеал $e_{i} F e_{i}$ кольиа е $e_{i} A e_{i}$ является вполне первичным.

(14) Пусть F - первичный идеал кольца A, причем все правые идеаль $\left(e_{i} A+F\right) / F$ кольца $A / F$ равномерны. Тогда

$F$ - несингулярно первичный идеал

$\Longleftrightarrow F-$ кусочно иелостный идеал

$\Longleftrightarrow$ для любого $e_{i} \in A \backslash F$ идеал $e_{i} F e_{i}$ кольиа $e_{i} A e_{i}$ является вполне первичным

$\Longleftrightarrow$ хотя бъц для одного $e_{i} \in A \backslash F$ идеал $e_{i} F e_{i}$ кольиа $e_{i} A e_{i}$ является вполне первичным.

ДокАЗАТЕльство. Пункт (1) проверяется непосредственно.

(2). Так как $e_{i} N e_{i}=0$ для всех $i$ и $N-$ правый идеал, то $N e_{i} N \subseteq N$ и $e_{j} N e_{i} N e_{j}=0$ для всех $i, j$. Тогда $N=\sum_{i \neq j} e_{i} N e_{j}$ и $N^{n}=\left(\sum_{i \neq j} e_{i} N e_{j}\right)^{\bar{n}}=0$.

(3). Пусть $h: A \rightarrow A / P$ - естественный эпиморфизм. Из леммы $3(2),(8)$ и из условия следует, что $h\left(e_{i}\right) h(N) h\left(e_{i}\right) \subseteq h\left(P_{i}\right)=h\left(e_{i}\right) h(P) h\left(e_{i}\right)=0$ для всех $i$. По примененному к $h(A)$ и $h(N)$ пункту $(2) h(N)$ - нильпотентный правьй идеал полупервичного кольца $h(A)$. Поэтому $h(N) \subseteq h(P), N \subseteq P$.

$(4),(5)$. Пункт (4) следует из (3) и его левостороннего аналога; (5) следует из (2) и из леммы $3(8)$.

(6). Пусть $B_{i} \equiv r\left(a e_{i}\right) \cap e_{i} A$. Если $a e_{i} \in \operatorname{Sing}\left(A_{A}\right)$, то $B_{i}$ - сушественньй подмодуль в $e_{i} A$, причем $a e_{i} B_{i}=0$. Необходимость доказана. Докажем достаточность. По условию найдется такой существенньй подмодуль $B_{i}$ модуля $e_{i} A$, что $B_{i} \subseteq r\left(a e_{i}\right)$. Пусть $D \equiv B_{i} \oplus \sum_{j \neq i} e_{j} A$. Тогда $D$ - сушественный правый идеал кольца $A$, лежащий в $r\left(a e_{i}\right)$. Поэтому $a e_{i} \in S$. 
$(7),(8),(9),(10),(11),(12)$. Пункт (7) следует из (6) и того, что $a=\sum_{i=1}^{n} a e_{i}$; (8) следует из (7); (9) и (12) проверяются непосредственно; (10) следует из (9); (11) следует из (10).

(13). По лемме $3(2)$ можно считать, что $F=0$ и $A$ - первичное кольцо. Импликация $\Longrightarrow$ очевидна. Докажем $\Longleftarrow$. Допустим, что сушествуют $e_{i}$ и ненулевые $a=a e_{i} \in A e_{i}, b=e_{i} b \in e_{i} A$ такие, что $a e_{i} b=0$. Для любого $d \in A$ правилом $f_{d}\left(e_{i} x\right)=e_{i} d a e_{i} x$ задается эндоморфизм $f_{d}$ модуля $e_{i} A$. Так как $a e_{i} b=0$, то все $f_{d}$ не являются мономорфизмами. По лемме $3(13)$ все эндоморфизмы $f_{d}$ являются нулевыми. Поэтому $e_{i} A a e_{i} A=0$. Так как $A$ - первично, то либо $e_{i} A=0$, либо $a A=a e_{i} A=0$. Получено противоречие. По (12) $A$ - кусочно целостное кольцо.

(14). По лемме $3(2)$ можно считать, что $F=0$ и $A$ - первичное кольцо. Первая эквивалентность вытекает из следующих фактов [4]: кусочно целостные кольца являются несингулярными; полуравномерные справа несингулярные справа кольца являются кусочно целостньми. Вторая эквивалентность следует из (13). Докажем третью эквивалентность. Импликация $\Longrightarrow$ очевидна. Докажем По (13) и по лемме $3(13)$ достаточно доказать, что для любого $j$ каждый эндоморфизм $f$ модуля $e_{j} A$, обладающий ненулевым ядром, тождественно равен нулю. Допустим, что $f\left(e_{j} A\right) \equiv N \neq 0$. Поскольку $A$ - первичное кольцо, то $N e_{i} A \neq 0$. Поэтому сушествует ненулевой гомоморфизм $h: e_{i} A \rightarrow N$. Поскольку $A$ первично, то $e_{i} A h\left(e_{i} A\right) \neq 0$. Поэтому сушествует ненулевой гомоморфизм $g: h\left(e_{i} A\right) \rightarrow e_{i} A$. Тогда $g h-$ ненулевой эндоморфизм модуля $e_{i} A$. Так как $e_{i} A-$ проективный модуль и $h\left(e_{i} A\right) \subseteq N \subseteq f\left(e_{j} A\right)$, то сушествует такой гомоморфизм $t: e_{i} A \rightarrow e_{j} A$, что $h=f t$. Тогда $t \neq 0$, поскольку $h \neq 0$. Так как $e_{j} A-$ равномерный модуль, то $M \equiv \operatorname{Ker}(f) \cap t\left(e_{i} A\right) \neq 0$. Поэтому $t^{-1}(M) \neq 0$, причем $(g h)\left(t^{-1}(M)\right)=(g f t)\left(t^{-1}(M)\right)=0$. По лемме $3(13) g h \equiv 0$, и получаем противоречие.

Лемма 5. Пусть $M_{R}-$ модуль над кольцом $R, \operatorname{End}(M) \equiv A, P-$ первичньи радикал кольча $A, N$ - правый идеал кольца $A$, причем $M_{R}=\bigoplus_{i=1}^{n} M_{i}$.

(1) Найдется такое разложение $1=e_{1}+\cdots+e_{n}$ единицы кольца $А$ в сумму ортогональных идемпотентов, что $M_{i}=e_{i}(M)$ и для любого $i$ существует естественныи кольцевой изоморфизм $\operatorname{End}\left(M_{i}\right) \cong e_{i} A e_{i}$.

(2) Пусть для любого $i$ естественный образ в кольце $\operatorname{End}\left(M_{i}\right)$ правого идеала е ${ }_{i} N e_{i}$ кольца $e_{i} A e_{i}$ лежит в первичном радикале $P_{i}$ кольца $\operatorname{End}\left(M_{i}\right)$. Тогда $N$ лехсит в $\mathrm{P}$.

(3) Пусть для любого $i$ первичный радикал кольца $\operatorname{End}\left(M_{i}\right)$ содерхсит все правые нильидеальи (все левье нильидеальь) кольца $\operatorname{End}\left(M_{i}\right)$. Тогда $P$ содерэст все правые нильидеаль (все левье нильидеаль) кольца $A$.

(4) Если для любого $i$ кольио $\operatorname{End}\left(M_{i}\right)$ полупервично, то $P^{n}=0$.

(5) Если $A$ - риккартово справа или слева кольцо и все кольца $\operatorname{End}\left(M_{i}\right)$ являются нормальными, то $P^{n}=0$.

ДокАЗАтЕльство. Пункт (1) проверяется непосредственно, а (2), (3) и (4) следуют из (1) и леммы 4. Докажем (5). По лемме $3(12)$ все кольца $\operatorname{End}\left(M_{i}\right)$ являются риккартовыми справа или слева нормальными кольцами. Тогда, как непосредственно проверяется, все кольца $\operatorname{End}\left(M_{i}\right)$ являются редуцированньги и, в частности, полупервичными. Теперь применяем (4). 
ПРЕДЛОЖЕНИЕ 1. Пусть $M_{R}-$ модуль, разлагающийся в конечную прямую сумму п нильпотентно-инвариантных модулей $M_{1}, \ldots, M_{n}, P$ - первичный радикал кольца $\operatorname{End}(M)$.

(1) Первичный радикал $P$ содержст все правые нильидеаль и все левъе нильидеальи кольца $\operatorname{End}(M)$.

(2) Если $M$ - несингулярныи модуль, то $P^{n}=0$ и все $\operatorname{End}\left(M_{i}\right)$ являются редуиированными кольцами.

(3) Если $M$ - несингулярный модуль и $\operatorname{End}(M)$ - первичное кольцо, то все кольиа $\operatorname{End}\left(M_{i}\right)$ являются областями .

ДокАЗАТЕЛЬСтво. Пункт (1) следует из лемм 1(6) и 5(3); (2) следует из лемм 2 и $5(4) ;(3)$ следует из лемм $2,3(10)$ и $5(1)$.

СлеДСТВИЕ 1. Пусть единица кольиа А является суммой таких ненулевых ортогональных идемпотентов $e_{1}, \ldots, e_{n}$, что все $e_{i} A$ - нильпотентно-инвариантные правые $A$-модули.

(1) Первичный радикал $P$ кольца $A$ содерэсит все правъе нильидеаль и все левье нильидеальи кольца $A$.

(2) Если $A$ - несингулярное справа кольцо, то $P^{n}=0$ и все кольиа $e_{i} A e_{i}$ являются редуцированными.

(3) Если $A$ - несингулярное справа первичное кольцо, то все кольца $e_{i} A e_{i}$ являются областями.

Следствие 1 вытекает из предложения 1.

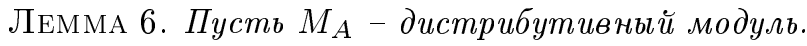

(1) Eсли $F, G \in \operatorname{Lat}(M)$ u $F \cap G=0, \operatorname{mo} \operatorname{Hom}(F, G)=0$.

(2) Кольио эндоморфизмов любого подфактора модуля $M$ является нормальнымм кольцом, причем $\operatorname{Hom}(F /(F \cap G), G /(F \cap G))=0$ для любъх подмодулей $F$ и $G$ модуля $M$.

(3) Eсли $F \in \operatorname{Lat}(M), f \in \operatorname{End}(M), n$ - натуральное число, причем $f^{n}(F) \subseteq F$, mo $f(F) \subseteq F$.

(4) Модуль $M$ - нильпотентно-инвариантный.

(5) Пусть все простые подфакторы модуля $M$ изоморфны (әто так, например, если факторкольцо $A / J(A)$ изоморфно кольиу матрии, над телом). Тогда $M-$ иепной модуль.

(6) Пусть $M$ обладает лишь конечным числом неизоморфных простых подфакторов (это так, например, если $A$ - полулокальное кольцо). Тогда М - конечномерный модуль.

ДокАЗАТЕЛьство. Пункт (1) доказан в предложении 1.1 из [6, §4.1]; (2) следует из (1) и дистрибутивности всех подфакторов модуля $M$.

(3). Индукция по $n$. При $n=1$ все ясно. Докажем утверждение для $n=2$. Пусть $G \equiv\{m \in M \mid f(m) \in F\}, h: M \rightarrow M /(F \cap G)$-естественный эпиморфизм и $f^{2}(F) \subseteq F$. Тогда $f(F) \subseteq G, h(F) \cap h(G)=0$, причем $f$ индуцирует гомоморфизм $\bar{f}: h(F) \rightarrow h(G)$. По $(1) \bar{f}(h(F))=0$. Поэтому $f(F) \subseteq F \cap G \subseteq F$. Пусть $n>2$ и утверждение верно для всех чисел, меньших $n$. Если $g \equiv f^{2}$, то $g^{n-1} \equiv 0$. По предположению индукции $f^{2}(F)=g(F) \subseteq F$. Так как для $n=2$ утверждение доказано, то $f(F) \subseteq F$. 
Пункт (4) следует из (3).

(5). Допустим противное. Тогда $M$ обладает подфактором $S \oplus T$, где $S$ и $T$ простые модули, которые по условию изоморфны. Так как $S \oplus T-$ дистрибутивный модуль, то получено противоречие с (1).

(6). Допустим противное. Тогда $M$ обладает подфактором $\bigoplus_{i=1}^{\infty} T_{i}$, где все $T_{i}-$ простые модули, среди которых по условию лишь конечное число неизоморфных. Поэтому модуль $M$ обладает подфактором $S \oplus T$, где $S$ и $T$ - изоморфные простые модули. Так как $S \oplus T$ - дистрибутивный модуль, то получено противоречие с (1).

ТЕОРема 1. Пусть $M_{A}$ - конечная прямая сумма $n$ дистрибутивнъх модулей $M_{1}, \ldots, M_{n}$.

Тогда первичный радикал $P$ кольца $\operatorname{End}(M)$ содержит все правье или левъе нильидеаль кольца $\operatorname{End}(M)$.

Если, кроме того, либо $M$ - несингулярный модуль, либо $\operatorname{End}(M)-$ риккартово справа или слева кольцо, то $P^{n}=0$.

Теорема 1 следует из предложения 1 , леммы 6(2) и леммы 5(5).

Примерами модулей $M_{A}$, к которым применима теорема 1 , являются кольца эндоморфизмов полуцепных модулей, кольца верхнетреугольных матриц над телами, любые факторкольца конечных прямых произведений колец матриц над дедекиндовыми кольцами и кольцо, построенное в замечании 6.

\section{§2. Полудистрибутивные кольца}

Лемма 7. Пусть $M-$ такой ненулевой правьй идеал кольца $A$, что $M_{A}-$ дистрибутивный модуль.

(1) Ecлu $m, n \in M$ u $m A \cap n A=0, m o m A n=0$.

(2) Если $A$ - первичное кольцо, то $M_{A}$ - равномерньй модуль.

ДокаЗательство. (1). Пусть $a \in A$. Правилом $f(n x)=\max$ задается гомоморфизм $f: n A \rightarrow m a A$. По лемме 6(1) $f \equiv 0$, man $=0$. Поэтому $m A n=0$; (2) следует из (1).

Лемма 8. Пусть единица кольца $А$ является суммой ненулевых ортогональных идемпотентов $e_{1}, \ldots, e_{n}$ и все правые $A$-модули $e_{i} A$ дистрибутивHbl.

(1) Если $A$ - первичное кольцо, то все $e_{i} A$ - равномерные правые $A$-модули и, следовательно, $A$ - полуравномерное справа кольцо, у которого правая размерность Голди равна п.

(2) Пусть $F$ - такой идеал кольца $A$, что $A / F$ - несингулярное справа первичное кольцо. Тогда для любого $e_{i} \in A \backslash F$ факторкольцо $e_{i} A e_{i} / e_{i} F e_{i}$ является областью.

(3) Пусть $A$ - первичное кольцо и все кольца е $_{i}$ Ае $e_{i}$ являются телами. Тогда все $e_{i} A$ - минимальные правые идеаль кольца $A$ и, следовательно, кольио $A$ изоморфно кольиу матрии размера $n \times n$ над телом.

(4) Если A - простое кольцо, то оно изоморфно кольцу матрии размера $n \times n$ над телом.

(5) Если $A$ - регулярное первичное кольчо, то А изоморфно кольиу матриц размера $n \times n$ над телом. 
(6) Пусть $B$ - идеал кольца $A, h: A \rightarrow A / B$ - естественный эпиморфизм. Тогда факторкольио $A / B$ является прямой суммой дистрибутивных правых идеалов $h\left(e_{i} A\right)$, некоторые из которых могут быть нулевыми.

ДокАЗАТЕЛЬство. Пункт (1) следует из леммы $7(2) ;(2)$ вытекает из следствия $1(3)$, лемм $3(2)$ и $6(4)$.

(3). Пусть $N=e_{i} N$ - ненулевой подмодуль в $e_{i} A, A_{i j} \equiv e_{i} A e_{j}$. Достаточно доказать, что $N e_{j}=A_{i j}$ для любого $j$. Из первичности кольца $A$ следует, что $N e_{j} \neq 0$. Из леммы 6(1) вытекает, что ненулевые дистрибутивные векторные пространства над телами являются одномерными. Поэтому из леммы 3(6) следует, что $A_{i j}$ - одномерное векторное пространство над телом $e_{j} A e_{j}$. Поэтому $0 \neq N e_{j}=A_{i j}$.

(4). Зафиксируем одно из колец $e_{i} A e_{i} \equiv R$. По (3) достаточно доказать, что $R-$ тело. По лемме $3(7),(10) R$ - дистрибутивное справа простое кольцо. Достаточно доказать, что произвольный максимальньй правый идеал $M$ кольца $R$ является идеалом. Допустим противное. Найдутся такие элементы $x \in R, m \in M$, что $x m \in R \backslash M$. Пусть $F \equiv\{a \in R \mid x a \in M\}, G \equiv\{a \in R \mid(1+x) a \in M\}$. Тогда $x \in R \backslash M$. Поэтому $F$ - максимальный правый идеал. Так как $x m \in R \backslash M$, то $m \in M \backslash F$. Поэтому $F \neq M, F+M=R$. Так как $(1+x) m-m=x m \in R \backslash M$, то $1+x \in R \backslash M$. Поэтому $G$ - максимальный правый идеал. Так как $(1+x) m \in R \backslash M$, то $m \in M \backslash G$. Поэтому $G \neq M, G+M=R$. Пусть $b \in F \cap G$. Тогда $b=(1+x) b-x b \in M$. Поэтому $F \cap G \subseteq M$. Из правой дистрибутивности кольца $R$ следует, что $R=(F+M) \cap(G+M)=F \cap G+M=M$. Получено противоречие.

Пункт (5) следует из (1) и из того, что конечномерное справа регулярное кольцо является артиновым; (6) следует из того, что $e_{i} B \subseteq B$ и, следовательно, $B=\bigoplus_{i=1}^{n}\left(B \cap e_{i} A\right)$.

Лемма 9. Для модуля $M_{A}$ над кольцом А равносильньц условия:

(1) $M-$ - дистрибутивный модуль;

(2) для любых әлементов $m, n$ модуля $M$ найдутся такие әлементы $a, b, c, d$ кольиа $A$, что $1=a+b, m a=n c, n b=m d$.

ДоказАтельство. (1) $\Rightarrow(2)$. Пусть $f=m+n, T \equiv m A \cap n A$. Так как $f A=f A \cap m A+f A \cap n A$, то найдутся такие элементы $b, d$ кольца $A$, что $f b \in m A$, $f d \in n A, f=f b+f d$. Тогда $n b=f b-m b \in T, m d=f d-n d \in T$. Пусть $a \equiv 1-b, z \equiv a-d=1-b-d$. Тогда $1=a+b, f z=f-f b-f d=0$. Поэтому $m a=m d+m z=m d+f z-n z=m d-n z$, причем $n z=-m z \in T$. Тогда $m a \in T$, откуда $a$ и $b$ - искомые элементы.

$(2) \Rightarrow(1)$. Пусть $F, G, H \in \operatorname{Lat}(M), f \in F \cap(G+H)$. Надо доказать включение $f \in F \cap G+F \cap H$. Пусть $f=m+n, m \in G, n \in H$. По условию найдутся такие элементы $a, b \in A$, что $1=a+b, m a \in n A, n b \in m A$. Тогда $f b=m b+n b \in$ $f A \cap m A \subseteq F \cap G, f a=m a+n a \in f A \cap n A \subseteq F \cap H, f=f b+f a \in F \cap G+F \cap H$.

Лемма 10. Пусть $F$ и $G$ - не сравнимые по включению вполне первичные подмодули дистрибутивного модуля $M_{A}$. Тогда $F+G=M$.

ДокаЗАТЕЛЬСтво. Пусть $h_{F}: M \rightarrow M / F$ и $h_{G}: M \rightarrow M / G$ - естественные эпиморфизмы, $f \in F \backslash G, g \in G \backslash F$. По лемме 9 найдутся такие элементы $a, b \in A$, 
что $1=a+b, f a=g c \in G, g b=f d \in F$. Тогда $a \in r\left(h_{G}(f)\right)=r\left(h_{G}(M)\right)$, откуда $M a \subseteq G$. Аналогично, $M b \subseteq F$. Поэтому $M=M a+M b \subseteq G+F \subseteq M$.

ПреДЛОЖЕНИЕ 2. Пусть $A$ - полудистрибутивное справа кольцо, разлагающееся в прямую сумму п дистрибутивных правых идеалов $e_{1} A, \ldots, e_{n} A$, где $e_{i}^{2}=e_{i} \in A$.

(1) Если $M$ - максимальный идеал кольца $A$, то факторкольцо $A / M$ изоморфно кольиу матрии над телом размера $m \times m$, где $m \leqslant n$.

(2) Если $B$ - пересечение некоторого множества максимальных идеалов кольца $A$, то индекс факторкольца $A / B$ не превосходит $n$.

(3) Пусть $F-$ первичный идеал кольча A. Тогда

$F$ - несингулярно первичный идеал

$\Longleftrightarrow F-$ кусочно иелостный идеал

$\Longleftrightarrow$ для любого $e_{i} \in A \backslash F$ идеал $e_{i} F e_{i}$ кольца е $e_{i} A e_{i}$ является вполне первичныцм

$\Longleftrightarrow e_{i} F e_{i}-$ вполне первичный идеал кольца $e_{i} A e_{i}$ хотя бы для одного $e_{i} \in A \backslash F$.

(4) Пусть все левые идеаль $А e_{i}$ дистрибутивны, $F$ - первичный идеал кольца $A$. Тогда $F$ - несингулярно первичный идеал $A / F$ - несингулярное слева кольцо.

(5) Пусть $F$ и $G$ - несингулярно первичные идеаль кольца A. Тогда либо $F+G=A$, либо идеалы $F$ и $G$ сравнимы по включению.

(6) Каждый собственный несингулярно первичный идеал F кольца $A$ содержит ровно один минимальный несингулярно первичный идеал.

ДокАЗАТЕЛЬСтво. Пункт (1) следует из леммы 8(4),(6).

(2). Так как индекс кольца матриц размера $m \times m$ над телом не превосходит $m$, то утверждение следует из (1) и из того, что класс колец, индекс которых не превосходит $m$, замкнут относительно перехода к подпрямьм произведениям.

(3), (4). Пункт (3) следует из лемм 4(14) и 7(2); (4) следует из (3).

(5). Допустим, что $F+G \neq A$. Найдется $e_{i} \equiv e \in A \backslash(F+G)$. По лемме $3(7)$ $e A e$ - дистрибутивное справа кольцо. По (3) $e F e, e G e$ - вполне первичные идеалы кольца $e A e$. Так как $e \in A \backslash(F+G)$, то $e A e \neq e F e+e G e$. Так как $e F e+$ $e G e \neq e A e$, то по лемме 10 можно считать, что вполне первичные идеалы $e F e, e G e$ дистрибутивного справа кольца $e A e$ сравнимы по включению. Поэтому можно без ограничения общности считать, что $e F e \subseteq e G e$. Так как $G$ - первичный идеал, причем $e \in A \backslash G$ и $e F e \subseteq G$, то $F \subseteq G$.

(6). В силу (5) и леммы Цорна достаточно доказать, что если $G=\bigcap_{j \in J} G_{j}-$ пересечение убьваюшей цепи лежащих в $F$ несингулярно первичных идеалов $G_{j}$, то $G$ - несингулярно первичный идеал. Так как все идеалы $G_{j}$ первичны, то $G-$ первичньй идеал. Пусть $e_{i} \equiv e \in A \backslash F$. По (3) все $e G_{j} e-$ вполне первичные идеалы кольца $e A e$. Тогда $e G e=\bigcap_{j \in J} e G_{j} e-$ вполне первичный идеал. По (3) $G$ - несингулярно первичный идеал.

СлеДСТвИЕ 2. Пусть A - полудистрибутивное справа регулярное кольцо, разлагающееся в прямую сумму п дистрибутивных правых идеалов.

(1) Пусть $M$ - первичный идеал кольца $A$. Тогда факторкольцо $A / M$ изоморфно кольиу матрич над телом размера $m \times m$, где $m \leqslant n, u, \boldsymbol{\theta}$ частности, $M$ - максимальный идеал. 
(2) Индекс любого факторкольца кольца $A$ не превосходит $n$.

(3) Максимальное правое кольцо частных $Q$ кольца $A$ является его максимальным левым кольцом частных и изоморфно конечному прямому произведению колеч матрич над строго регулярными кольцами.

ДокАЗАТЕльство. Пункт (1) следует из леммы 8(5),(6); (2) следует из предложения 2(2) и того, что любой идеал регулярного кольца является пересечением первичных идеалов; (3) следует из (2) и того, что утверждение пункта (3) верно для любого регулярного кольца ограниченного индекса (см. [7, теоремы 7.18, 7.20 и следствие 7.4]).

ЛЕМма 11. Пусть единица кольца $А$ является суммой ортогональньх идемпотентов $e_{1}, \ldots, e_{n}$, причем все кольца $e_{i} A e_{i}$ дистрибутивны справа.

(1) Пусть $F$ и $G$ - не сравнимые по включению первичные идеаль кольца $A$, причем для любого $i$ кольца $e_{i} A e_{i} / e_{i} F e_{i}$ и $e_{i} A e_{i} / e_{i} G e_{i}$ являются либо областями, либо нулевыми кольцами. Тогда $F+G=A$.

(2) Пусть $F$ и $G$ - не сравнимые по включению несингулярно первичные идеаль кольиа $A$. Тогда $F+G=A$.

(3) Первичный радикал $P$ кольиа $A$ содержст все правые нильидеаль и все левье нильидеальи кольиа $A$.

ДоказАтельство. (1). Допустим противное. Так как $1 \in A \backslash(F+G)$, то найдется идемпотент $e_{i} \in A \backslash(F+G)$. Из условия и из леммы $3(11)$ следует, что $e_{i} F e_{i}$, $e_{i} G e_{i}-$ не сравнимые по включению вполне первичные идеалы дистрибутивного справа кольца $e A e$ и $e F e+e G e \neq e A e$. Получено противоречие с леммой 10.

(2). Зафиксируем идемпотент $e_{i}$. По (1) достаточно доказать, что $e_{i} A e_{i} / e_{i} F e_{i}$ является либо областью, либо нулевьм кольцом. Пусть $e_{i} A e_{i} / e_{i} F e_{i} \neq 0$. Тогда $e_{i} \in A \backslash F$. Пусть $h: A \rightarrow A / F$ - естественный эпиморфизм. По лемме 8(2) $e_{i} A e_{i} / e_{i} F e_{i}$ - область.

(3). Зафиксируем идемпотент $e_{i}$. Пусть $P_{i}$ - первичный радикал кольца $e_{i} A e_{i} \equiv R_{i}$. Так как $R_{i}$ - дистрибутивное справа кольцо, то по теореме 1 все правые или левые нильидеалы кольца $R_{i}$ лежат в $P_{i}$. По лемме $4(4) P$ содержит все правые или левые нильидеалы кольца $A$.

ЛЕмма 12. Пусть $R$ - полупервичное кольцо с условием максимальности для аннуляторных идеалов.

(1) Существуют такие минимальные первичные идеальи $F_{1}, \ldots, F_{n}$ кольиа $A$, что $F_{1} \cap \cdots \cap F_{n}=0$, но никакое пересечение менее чем $n$ идеалов $F_{i}$ не равно нулю.

(2) Если $R$ - несингулярное справа кольцо, то все $A / F_{i}$ - несингулярные справа кольца.

ДоКАЗАТЕЛЬСТво. Пункт (1) следует из того, что $R$ - подпрямое произведение конечного числа первичных колец [5, с. 273, 274].

(2). Пусть $F_{1}, \ldots, F_{n}$ - минимальные первичные идеалы из пункта (1). Будем считать, что $n>1$, поскольку при $n=1$ утверждение очевидно. Пусть $F \equiv F_{1}, B \equiv F_{2} \cap \cdots \cap F_{n} \neq 0, h: A \rightarrow A / F-$ естественный эпиморфизм. Достаточно доказать, что $h(A)$ - несингулярное справа кольцо. Пусть $D$ - такой правый идеал кольца $A$, что $D$ содержит идеал $F$ и $h(B) \cap h(D)=0$. Тогда 
$h(D) h(B) \subseteq H(D) \cap h(B)=0$. Так как $h(A)$ - первичное кольцо, то $h(D)=0$. Поэтому $h(B)$ - существенный правый идеал кольца $h(A)$. Тогда достаточно доказать, что $h(B)_{h(A)}$ - несингулярный модуль. Пусть $b$ - такой элемент идеала $B$, что $r_{h(A)}(h(b))$ - существенный правый идеал кольца $h(A), E$ - такой правый идеал кольца $A$, что $h(E)=h(B) \cap r_{h(A)}(h(b))$ и $F \subseteq E \subseteq B \oplus F$. Так как $h(B)$ существенный правый идеал кольца $h(A)$, то $h(E)$ - существенный правый идеал кольца $h(A)$. Поэтому $E$ - сушественный правый идеал, причем $b E \subseteq B \cap F=0$. Так как $A$ - несингулярное справа кольцо, то $b=0, h(b)=0$.

Лемма 13. Пусть $F_{1}, \ldots, F_{n}-$ такие идеальи кольца $A$, ито $F_{1} \cap \cdots \cap F_{n}=0$ u $F_{i}+F_{j}=A$ для всех $i \neq j$. Тогда $A \cong \prod_{i=1}^{n} A / F_{i}$.

Лемма 13 ("Китайская теорема об остатках") хорошо известна и проверяется непосредственно. При доказательстве используется следуюшее замечание: если $F, G, H$ - идеалы кольца $A$ и $F+G=F+H=A$, то $A=F+G \cap H$, поскольку $A=(F+G)(F+H) \subseteq F+G H$.

Лемма 14 [8, с. 56, лемма 2.5]. Если $A$ - кольцо с условием максимальности для правых аннуляторов, то $\operatorname{Sing}\left(A_{A}\right)$ - нильпотентный идеал.

ЛЕмма 15. Пусть $A$ - полупервичное кольцо, удовлетворяющее либо условию максимальности для правых аннуляторов, либо условию максимальности для левых аннуляторов. Тогда $A$ - несингулярное кольцо.

ДокАЗАТЕЛЬСтво. Достаточно рассмотреть случай условия максимальности для левых аннуляторов. Левая несингулярность кольца $A$ следует из леммы 13 . Допустим, что правый сингулярньй идеал $B$ кольца $A$ не равен нулю. Кольцо $A$ удовлетворяет условию минимальности для правых аннуляторов. Поэтому найдутся такие элементы $b_{1}, \ldots, b_{n} \in B$, что $r(B)=r\left(b_{1}, \ldots, b_{n}\right)=r\left(b_{1}\right) \cap \cdots \cap r\left(b_{n}\right)$. Тогда $r(B)$ - сушественный правый идеал, поскольку все правые идеалы $r\left(b_{i}\right)$ являются сушественньми. Поэтому $D \equiv B \cap r(B) \neq 0$ и $D^{2}=0$, что противоречит полупервичности кольца $A$.

Лемма 16 (см. [9, лемма 9.12]). Пусть $A$ - конечномерное справа полупер-

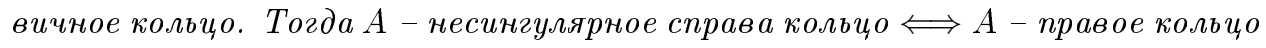
Голди.

ПрЕДЛОЖЕНИЕ 3. Пусть $A$ - полудистрибутивное справа несингулярное справа полупервичное кольцо с условием максимальности для аннуляторных идеалов. Тогда $A$ - конечное прямое произведение первичных правых колеи Голди $A_{1}, \ldots, A_{n}$, причем каждое кольио $A_{i}$ разлагается в конечную прямую сумму таких дистрибутивных равномерных правых идеалов $e_{i 1} A, \ldots, e_{i m(i)} A$, что $e_{i j}{ }^{2}=e_{i j}$, а все кольца $e_{i j(i)} A_{i} e_{i j(i)}$ являются дистрибутивньми справа областями.

ДокАЗАТЕЛЬСтво. По лемме 12 существуют такие минимальные первичные идеалы $F_{1}, \ldots, F_{n}$ кольца $A$, что $F_{1} \cap \cdots \cap F_{n}=0$ и все $A / F_{i}$ - несингулярные справа первичные кольца. По лемме $8(1)$ все $A / F_{i}$ - конечномерные справа кольца, являющиеся по лемме 16 правьми кольцами Голди. По лемме $11(2) F_{i}+F_{j}=A$ для всех $i \neq j$. По лемме $12 A \cong \prod_{i=1}^{n} A / F_{i}$. Теперь к первичным кольцам $A / F_{i}$ применяем лемму $8(1)$. 
СЛЕДСТВИЕ 3. Пусть $A$ - полудистрибутивное справа кольцо, являющееся подпрямым произведением конечного числа первичных колец $A_{1}, \ldots, A_{n}$. Тогда $A$ - конечномерное справа полупервичное кольио. Следовательно, если $A$ - несингулярное справа кольцо, то $A$ - правое кольио Голди.

ДокАЗАтЕльство. Так как по лемме $8(1)$ все кольца $A_{i}$ конечномерны справа, причем подпрямое произведение конечного числа конечномерных справа колец является конечномерным справа кольцом, то $A$ - конечномерное справа кольцо. Кроме того, подпрямое произведение первичных колец является полупервичным кольцом. Далее применим лемму 16.

Теорема 2. Пусть $A$ - полудистрибутивное справа полупервичное кольцо либо с условием максимальности для правых аннуляторов, либо с условием максимальности для левых аннуляторов.

Тогда $A$ - конечное прямое произведение первичных правых колеи, Голди, разлагающихся в конечную прямую сумму дистрибутивных равномерных правых идеалов.

Теорема 2 следует из предложений 1(3), 3 и леммы 15.

Примерами колец $A$, к которым применима теорема 2 , являются конечные прямые произведения колец матриц над дедекиндовыми кольцами (например, над кольцами целых алгебраических чисел).

СлЕДСТВИЕ 4. Полудистрибутивное справа полупервичное кольцо, обладающее правой или левой размерностью Крулля, разлагается в конечное прямое произведение несингулярных первичных колеи.

Следствие 4 вытекает из теоремы 2 , леммы 15 и того, что полупервичное кольцо с правой (левой) размерностью Крулля является правым (левым) кольцом Голди [10, следствие 3.4$]$.

СлЕДСТВИЕ 5. Для первичного идеала $M$ полудистрибутивного справа кольца А равносильны условия:

(1) $M-$ - несингулярно первичный идеал;

(2) $A / M$ - кольцо с условием максимальности для правых аннуляторов;

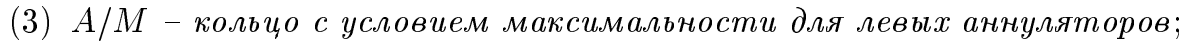

(4) $A / M-$ правое кольцо Голди.

В следствии 5 импликации $(3) \Rightarrow(1)$ и $(2) \Rightarrow(1)$ вытекают из леммы 15 , импликация $(1) \Rightarrow(4)$ - из следствия 3 , а импликации $(4) \Rightarrow(3)$ и $(4) \Rightarrow(2)$ - из того, что по теореме Голди первичное правое кольцо Голди является правым порядком в простом артиновом кольце.

Замечание 1 . Пусть единица кольца $A$ разлагается в сумму таких ненулевых ортогональных идемпотентов $e_{1}, \ldots, e_{n}$, что все правые идеалы $e_{i} A$ равномерны.

(1) $\operatorname{Sing}\left(A_{A}\right)$ совпадает со множеством всех элементов $a \in A$, таких что для любого $e_{i}$ найдется такой ненулевой элемент $b \in e_{i} A$, что $a b=0$.

(2) Пусть $i \in\{1, \ldots, n\}, a \in A e_{i}$. Тогда $a \in \operatorname{Sing}\left(A_{A}\right) \Longleftrightarrow$ сушествует такой ненулевой элемент $b \in e_{i} A$, что $a b=0$.

(3) $A$ - несингулярное справа кольцо $\Longleftrightarrow$ для любого идемпотента $e_{i}$ верно, что $a b \neq 0$ для каждого ненулевого элемента $a=a e_{i} \in A e_{i}$ и для любого ненулевого элемента $b=e_{i} b \in e_{i} A$. 
(4) $A$ - несингулярное справа кольцо $\Longleftrightarrow$ для любых идемпотентов $e_{i}, e_{j}, e_{k}$ верно, что $a b \neq 0$ для каждого ненулевого элемента $a=e_{j} a e_{i} \in e_{j} A e_{i}$ и для любого ненулевого элемента $b=e_{i} b e_{k} \in e_{i} A e_{k}$.

(5) Пусть $A$ - кольцо с условием максимальности для правых аннуляторов, $a \in A e_{i}$ и сушествует такой ненулевой элемент $b \in e_{i} A$, что $a b=0$. Тогда идеал кольца $A$, порожденный элементом $a$, является нильпотентным.

Пункт (1) замечания 1 вытекает из леммы 4(7), равенств $a=a e_{i}, b=e_{i} b$ и того, что все ненулевые подмодули равномерного модуля являются сушественными; (2) следует из (1); (3) следует из (1) и из леммы $4(8) ;(4)$ вытекает из (3) и того, что если $a e_{i} \neq 0, e_{i} b \neq 0$, то $e_{j} a e_{i} \neq 0, e_{i} b e_{k} \neq 0$ для некоторых $e_{j}, e_{k} ;(5)$ следует из (2) и из леммы 14.

ЗАмечание 2 . Пусть единица кольца $A$ разлагается в сумму таких ненулевых ортогональных идемпотентов $e_{1}, \ldots, e_{n}$, что все правые идеалы $e_{i} A$ и все левые идеалы $A e_{i}$ равномерны. Равносильны условия:

(1) $A$ - несингулярное справа кольцо;

(2) $A$ - несингулярное слева кольцо;

(3) для любых идемпотентов $e_{i}, e_{j}, e_{k}$ верно, что $a b \neq 0$ для каждого ненулевого элемента $a=e_{j} a e_{i} \in e_{j} A e_{i}$ и для любого ненулевого элемента $b=e_{i} b e_{k} \in e_{i} A e_{k}$.

Замечание 2 вытекает из замечания 1(3),(4) и симметричности условия (3).

ЛЕмма 17. Пусть $1=\sum_{i=1}^{n} e_{i}-$ разложсение единицы полусовериенного кольца $А$ в сумму локальных ортогональных идемпотентов.

(1) Если $M$ - правый А-модуль, то дистрибутивность модуля $M$ равносильна тому, что $\mathrm{Mе}_{i}$ является цепныцм правым модулем над кольцом $e_{i} A e_{i}$ для любого $e_{i}$.

(2) Правая полудистрибутивность кольца А равносильна тому, что е ${ }_{j} A e_{i}$ является цепныц правым модулем над кольцом е Ае $_{i}$ для любых е $e_{i}$ и е

Пункт (1) леммы 17 следует из лемм 4(11) и 6(5); (2) следует из (1).

ЗАмечание 3 . Из теоремы 1 вытекает, что первичный радикал $P$ полудистрибутивного справа кольца $A$ содержит все правые или левые нильидеалы кольца $A$, причем если $A$ - несингулярное справа кольцо, то $P$ - нильпотентный идеал.

ЗАмЕчАниЕ 4. Кольцо матриц второго порядка над кольцом целых чисел дает пример первичного нётерова полудистрибутивного PI-кольца, не являющегося ни полуцепным справа, ни дистрибутивным справа.

При анализе возможных гипотез о строении полудистрибутивных колецполезно иметь в виду замечание 5 .

ЗАмечАниЕ 5 . Существует такая 5-мерная алгебра $A$ над полем $F$ с $F$-базисом $\left\{e_{11}, e_{12}, e_{13}, e_{22}, e_{33}\right\}$, что

(1) $e_{11}, e_{22}, e_{33}$ - локальные ортогональные идемпотенты, $1=e_{11}+e_{22}+e_{33}$;

(2) $e_{22} A$ и $e_{33} A$ - простые проективные правые $A$-модули, изоморфные модулям $e_{12} A$ и $e_{13} A$, соответственно;

(3) ${ }_{A} A=A e_{11} \oplus A e_{22} \oplus A e_{33}$, где $A e_{11}$ - простой проективный левьй $A$-модуль, а $A e_{22}$ и $A e_{33}$ - цепные левые $A$-модули, являюшиеся сушественными расширениями простых неизоморфных модулей $A e_{12}$ и $A e_{13}$, соответственно; 
(4) $e_{11} A$ - неразложимый неравномерный проективный дистрибутивный локальный $A$-модуль, у которого все собственные ненулевые подмодули исчерпываются простыми проективньпи неизоморфными модулями $e_{12} A, e_{13} A$ и полупростым проективным модулем $e_{12} A \oplus e_{13} A$;

(5) $e_{12} A$ - замкнутый подмодуль дистрибутивного модуля $e_{11} A$, не являюшийся прямым слагаемым модуля $e_{11} A$;

(6) каждый неразложимьй инъективный правый $A$-модуль является циклическим цепным модулем;

(7) каждый неразложимый правый $A$-модуль является либо простым модулем, либо циклическим инъективным цепным модулем, либо циклическим проективным дистрибутивньм модулем;

(8) $A$ - полудистрибутивная справа полуцепная слева наследственная конечномерная $F$-алгебра (и, в частности, несингулярное полудистрибутивное кольцо), не являющаяся полуравномерным справа кольцом, причем каждый правый $A$-модуль является полудистрибутивным и разлагается в прямую сумму полупростого модуля, инъективного полуцепного модуля и проективного полудистрибутивного модуля.

Нужный пример дает подалгебра $A$ алгебры всех верхнетреугольных матриц третьего порядка над полем $F$, у которой $F$-базисом служат матричные единицы $\left\{e_{11}, e_{12}, e_{13}, e_{22}, e_{33}\right\}$. Доказательство замечания 5 может быть проведено элементарными рассуждениями и стандартньми методами теории конечномерных алгебр над полем.

ЗАмЕчАнИЕ 6. В утверждениях 2.4 и 2.5 [3] делается попытка доказать следующее: если $M_{A}$ - дистрибутивньй несингулярный конечномерный модуль над полудистрибутивным справа полусовершенным кольцом $A$, то $M$ - полуравномерный модуль, у которого каждый замкнутый подмодуль выделяется прямьм слагаемым. В следствии 2.6 [3] утверждается, что полудистрибутивное справа несингулярное справа полусовершенное кольцо является полуравномерным справа. Из замечания 6 (см. пункты (4), (5) и (8)) следует, что все эти утверждения не верны. Ошибка содержится в последнем предложении доказательства утверждения 2.4 [3].

ЛЕмма 18. Каждый модуль над полулокальным кольцом, разлагающийся в конечную прямую сумму дистрибутивных модулей, является конечномерным модулем.

Лемма 18 вытекает из леммы 6(6) и того, что конечная прямая сумма конечномерных модулей является конечномерньм модулем.

Лемма 19. Пусть $A$ - полудистрибутивное справа полулокальное кольцо.

(1) Каждый ииклический правый А-модуль является конечномерным модулем.

(2) Кажсдое несингулярное справа факторкольцо кольца А является правым кольцом Голди с условием максимальности для левых аннуляторов.

ДоказАтЕльство. Пункт (1) следует из леммы 18. Так как подкольцо кольца с условием максимальности для правых (левых) аннуляторов само обладает этим свойством, то пункт (2) следует из пункта (1) и того, что максимальное правое кольцо частных несингулярного справа конечномерного справа кольца - полупростое артиново. 
Лемма 20. Пусть $A$ - полупервичное кольио.

(1) Пусть существует идеал $B$ кольца $A$, разлагающийся в прямую сумму идеалов $M_{1}, \ldots, M_{n}$ кольца $A$, причем $r(B)=0$, и все идеаль $r\left(M_{i}\right)$ являются первичными идеалами кольиа $A$. Тогда $A$ - подпрямое произведение конечного числа первичных колеч, и удовлетворяет условию максимальности для аннуляторных идеалов.

(2) Если ненулевой идеал $M$ кольца $A$ не содержит прямой суммы никаких двух ненулевых идеалов кольца $A$, то $r(M)$ - первичный идеал.

Пункт (1) леммы 20 следует из [5, 1 гл. 4, теорема 3 и лемма 4]; (2) проверяется непосредственно.

Лемма 21. Пусть $A$ - полупервичное кольио, не содержащее бесконечных прямых сумм ненулевых идеалов.

(1) $A$ - подпрямое произведение конечного числа первичных колец и удовлетворяет условию максимальности для аннуляторных идеалов.

(2) Пусть сумма любых двух не сравнимых по включению первичных идеалов кольиа А равна $A$. Тогда $A$ - конечное прямое произведение первичньцх колец.

Пункт (1) леммы 21 следует из леммы 20; (2) следует из (1) и леммы 13.

Лемма 22. Пусть $A-$ полупервичное РІ-кольио.

(1) $A$ - несингулярное кольцо.

(2) Если A - полудистрибутивное справа кольцо с условием максимальности для аннуляторных идеалов, то $A$ - конечное прямое произведение первичных несингулярных колеи.

Пункт (1) леммы 22 доказан, например, в [11, следствие 6.1.31]; (2) следует из (1) и предложения 2.

ПрЕДЛОЖЕНИЕ 4. Пусть единица кольца $A$ является суммой таких ненулевых ортогональных идемпотентов $e_{1}, \ldots, e_{n}$, что во всех кольцах $e_{i}$ Ае любые два первичных идеала либо сравнимы по включению, либо дают в сумме кольцо е ${ }_{i} A e_{i}$. (Это так, например, если решетка идеалов кажсдого кольца $e_{i} A e_{i}$ является иепью.)

(1) Сумма любых двух не сравнимых по включению первичных идеалов кольиа А равна $A$.

(2) Пусть $A$ - полупервичное кольцо, не содержащее бесконечных прямых сумм ненулевых идеалов. Тогда $A$ - конечное прямое произведение первичных колец.

(3) Пусть $A$ - полупервичное кольцо, не содержащее бесконечных прямых сумм ненулевых идеалов. Тогда $A$ - кусочно иелостное относительно $\left\{e_{1}, \ldots, e_{n}\right\}$ кольцо $\Longleftrightarrow e_{i} A e_{i}-$ область для любого $e_{i}$.

(4) Пусть $F$ - полупервичный идеал кольца $A$, причем факторкольцо $A / F$ не содерэит бесконечных прямых сумм ненулевых идеалов. Тогда $F$ кусочно челостный относительно $\left\{e_{1}, \ldots, e_{n}\right\}$ идеал $\Longleftrightarrow e_{i} F e_{i}-$ вполне первичный идеал кольиа $e_{i} A e_{i}$ для любого $e_{i} \in A \backslash F$. 
(5) Пусть $F$ - полупервичный идеал кольца $A$, причем факторкольио $A / F$ не содержит бесконечных прямых сумм ненулевых идеалов и все правые идеальь $\left(e_{i} A+F\right) / F$ кольца $A / F$ равномерньл. Тогда

$F$ - несингулярно полупервичный идеал

$\Longleftrightarrow F-$ кусочно иелостныи относительно $\left\{e_{1}, \ldots, e_{n}\right\}$ идеал

$\Longleftrightarrow$ для любого $e_{i} \in A \backslash F$ идеал $e_{i} F e_{i}$ кольиа $e_{i} A e_{i}$ является вполне первичным.

ДокАЗАтЕльство. Пункт (1) следует из леммы 3(11) и следующего факта: если $F$ и $G$ - такие идеалы кольца $A$, что $F+G \neq A$, то идеал $F+G$ не содержит хотя бы один идемпотент $e_{i} ;(2)$ следует из $(1)$ и из леммы $21 ;(3)$ следует из $(2)$ и леммы 4(13); (4) следует из (3) и леммы $3(3) ;(5)$ следует из (4) и леммы 4(14).

ПРЕДЛОЖЕНИЕ 5. Пусть $A$ - полудистрибутивное справа полусовершенное кольцо, $1=\sum_{i=1}^{n} e_{i}-$ разложсение единищъ кольца $A$ в сумму локальных идемпотентов.

(1) Сумма любъх двух не сравнимых по включению первичных идеалов кольча А равна $A$.

(2) Если $A$ - полупервичное кольцо, то $A$-конечное прямое произведение первичных колеи.

(3) Пусть $F$ - полупервичный идеал кольца А. Тогда

$F$ - несингулярно полупервичный идеал

$\Longleftrightarrow F-$ кусочно иелостный относительно $\left\{e_{1}, \ldots, e_{n}\right\}$ идеал

$\Longleftrightarrow$ для любого $e_{i} \in A \backslash F$ идеал $e_{i} F e_{i}$ кольиа $e_{i} A e_{i}$ является вполне первичнымм

$\Longleftrightarrow A / F-$ правое кольио Голди.

ДоказАТЕльство. По лемме 17(2) кольцо $A$ удовлетворяет условиям предложения 4. По лемме 19 каждое факторкольцо кольца $A$ является конечномерным справа кольцом. Поэтому утверждение следует из предложения 4 и леммы 16.

ПрЕДЛОЖЕНИЕ 6. Пусть $A$ - полудистрибутивное справа полусовершенное кольцо, $1=\sum_{i=1}^{n} e_{i}-$ разложсение единиць кольиа $A$ в сумму локальных идемпотентов, $N$ - пересечение всех несингулярно полупервичных идеалов кольиа A. Тогда справедливь следующие утверждения.

(1) $N$ - наименьиий несингулярно полупервичный идеал кольца $A$.

(2) $N$ - наименьиий кусочно целостный относительно $\left\{e_{1}, \ldots, e_{n}\right\}$ полупервичный идеал кольца $A$.

(3) $N$ - наименьший идеал кольца $A$, факторкольцо кольца $A$ по которому является полупервичным правым кольцом Голди.

(4) Пусть кольио А либо обладает правой или левой размерностью Крулля, либо является РІ-кольцом. Тогда идеал $N$ совпадает с первичным радикалом кольиа $A$.

ДоКАЗАТЕЛЬСтво. Пусть $\mathscr{E}-$ множество всех несингулярно полупервичных идеалов.

(1), (2), (3). По предложению 5(3) множество $\mathscr{E}$ совпадает как со множеством всех кусочно целостных относительно $\left\{e_{1}, \ldots, e_{n}\right\}$ полупервичных идеалов, так 
и со множеством всех таких идеалов кольца $A$, факторкольца кольца $A$ по которым являются полупервичными правыми кольцами Голди. Поэтому достаточно доказать, что $N$ - несингулярно полупервичный идеал. Так как $N$ - пересечение некоторого множества полупервичных идеалов кольца $A$, то $N$ - полупервичньй идеал. Пусть $e_{i} \in A \backslash N$. По предложению 5(3) достаточно доказать, что $e_{i} N e_{i}$ - вполне первичный идеал кольца $e_{i} A e_{i}$. Пусть $\mathscr{E}=\left\{F_{j}\right\}_{j \in J}$. Тогда $e_{i} N e_{i}=e_{i}\left(\bigcap_{j \in J} F_{j}\right) e_{i}=\bigcap_{j \in J}\left(e_{i} F_{j} e_{i}\right)$. По предложению 5(3) все идеалы $e_{i} F_{j} e_{i}$ кольца $e_{i} A e_{i}$ являются вполне первичными. По лемме $17(2) e_{i} A e_{i}$ - цепное справа кольцо. Поэтому $e_{i} N e_{i}-$ пересечение цепи вполне первичных идеалов $e_{i} F_{j} e_{i}$ кольца $e_{i} A e_{i}$. Тогда $e_{i} N e_{i}-$ вполне первичньй идеал кольца $e_{i} N e_{i}$.

(4). Пусть $F$ - полупервичный идеал кольца $A$. Достаточно доказать, что $A / F$ - несингулярное справа кольцо. Если $A$ обладает правой или левой размерностью Крулля, то это вытекает из следствия 4. Каждьй полупервичньй идеал кольца $A$ является несингулярно полупервичньм.

СЛЕДСТВИЕ 6. Пусть $A$ - полудистрибутивное справа несингулярное справа полупервичное кольцо, причем кольцо $A$ либо не содержит бесконечных прямых сумм ненулевых идеалов, либо является полулокальным кольцом. Тогда $A$ - конечное прямое произведение полуравномерных справа первичных правых колеи Голди.

Следствие 6 вытекает из предложения 3, а также лемм 19 и 21.

Лемма 23. Пусть $M_{A}-$ проективный модуль над несингулярным справа кольиом $A$.

(1) Если $M$ - существенное расширение конечнопорожденного модуля $N$, то $M-$ конечнопорожсденный модуль.

(2) Если $M$ - конечномерный модуль, то $M$ - конечнопорожсденный модуль.

ДокАЗАТЕЛьство. Доказывать надо только (1), поскольку каждый конечномерный модуль является существенным расширением конечной прямой суммы циклических равномерных модулей. Так как $M$ - проективный модуль, то найдутся такие прямые суммы $M \oplus L=\bigoplus_{j \in J} G_{j} \equiv F$, что $G_{j} \cong A_{A}$ для всех $j$. Поскольку $N$ - конечнопорожденный модуль, то найдется такое конечное множество $D \subseteq J$, что $N \subseteq \bigoplus_{j \in D} G_{j} \equiv H$. Пусть $f: F \rightarrow \bigoplus_{j \in J \backslash D} G_{j}$ - проекция с ядром $H$. Несингулярность модуля $A_{A}$ влечет несингулярность свободного модуля $F$. Так как $f(N)=0$ и $M-$ существенное расширение модуля $N$, то $f(M) \subseteq \operatorname{Sing}(F)=0$. Поэтому $M \subseteq H$. Тогда прямое слагаемое $M$ модуля $F$ является прямым слагаемым конечнопорожденного модуля $H$. Поэтому $M$ - конечнопорожденньй модуль.

ЛЕмма 24. Риккартово справа кольцо является несингулярным справа.

Лемма 24 проверяется непосредственно.

Лемма 25. Пусть А - наследственное справа кольцо. Тогда каждый конечномерный правый идеал кольца $A$ является конечнопорожсденым. $B$ частности, если $A$ - конечномерное справа кольцо, то $A$ - нётерово справа кольцо.

Лемма 25 вытекает из лемм 23(2) и 24. 
ЛЕмма 26. Наследственное справа полудистрибутивное справа полулокальное кольцо является нётеровым справа кольцом.

Лемма 26 следует из лемм 19 и 25.

ТЕОРема 3. Пусть А - наследственное справа полудистрибутивное справа полупервичное кольцо.

(1) Если А не содерэсит бесконечных прямых сумм ненулевых идеалов, то $A$ - конечное прямое произведение нётеровых справа первичных колеи.

(2) Если $A$ - полулокальное кольцо, то $A$ - конечное прямое произведение нётеровых справа первичных колеч.

ДоказАТЕЛЬСтво. (1). По лемме $23 A$ - несингулярное справа кольцо. По следствию 6 можно без ограничения обшности считать, что $A$ - полуравномерное справа первичное кольцо. Полуравномерное справа кольцо $A$ является конечномерным справа. По лемме $24 A$ - нётерово справа кольцо.

Пункт (2) следует из (1) и из леммы 25.

Примерами колец $A$, к которым применима теорема 3 , являются наследственные полуцепные полупервичные кольца (например, конечные прямые произведения колец матриц над кольцами формальных степенных рядов от одной переменной над полями). Другие примеры таких колец приведены, например, в [12].

\section{Список литературы}

1. Туганбаев A. А. Колњц с плоскими правыми идеалами и дистрибутивные колњца // Матем. заметки. 1985. Т. 38. № 2. С. 218-228.

2. Mazurek R., Puczylowski E. On nilpotent elements of distributive rings // Comm. Algebra. 1990. V. 18. № 2. P. 463-471.

3. Wright Mary H. Right locally distributive rings // Ring Theory (Granville, OH, 1992). River Edge, NJ: World Sci. Publ., 1993. P. 350-357.

4. Gordon R., Small L. W. Piecewise domains // J. Algebra. 1972. V. 23. № 3. P. 553-564.

5. Андрунакиевич B. А., Рябухин Ю. М. Радикалы алгебр и структурная теория. М.: Наука, 1979.

6. Кон П. Свободные кольца и их связи. М.: Мир, 1975.

7. Goodearl K. R. Von Neumann regular rings. London: Pitman, 1979.

8. Stenström B. Rings of quotients: an introduction to methods of ring theory. Berlin: Springer-Verlag, 1975.

9. Фейс K. Алгебра: колцца, модули и категории. Т. 1. М.: Мир, 1977.

10. Gordon R., Robson J. C. Krull dimension // Mem. Amer. Math. Soc. 1973. V. 133. P. 1-78.

11. Rowen L. H. Ring theory. Student edition. Boston: Academic Press, 1991.

12. Кириченко В. В. Обобщенно однорядные кольца // Матем. сб. 1976. Т. 99. № 4. C. 559-581. 